PROCEEDINGS OF THE

AMERICAN MATHEMATICAL SOCIETY

Volume 138, Number 7, July 2010, Pages 2341-2347

S 0002-9939(10)10289-5

Article electronically published on March 4, 2010

\title{
ON THE DEGREE OF RAPID DECAY
}

\author{
BOGDAN NICA \\ (Communicated by Varghese Mathai)
}

\begin{abstract}
A finitely generated group $\Gamma$ equipped with a word-length is said to satisfy property RD if there are $C, s \geq 0$ such that, for all non-negative integers $n$, we have $\|a\| \leq C(1+n)^{s}\|a\|_{2}$ whenever $a \in \mathbb{C} \Gamma$ is supported on elements of length at most $n$.

We show that, for infinite $\Gamma$, the degree $s$ is at least $1 / 2$.
\end{abstract}

\section{INTRODUCTION}

Let $\Gamma$ be a finitely generated group, and fix a word-length on $\Gamma$. We say that $\Gamma$ has property $R D$ if there exist $C, s \geq 0$ such that, for all integers $n \geq 0$, we have $\|a\| \leq C(1+n)^{s}\|a\|_{2}$ whenever $a \in \mathbb{C} \Gamma$ is supported on elements of length at most $n$. Here $\|\cdot\|$ denotes the operator norm coming from the regular representation of $\Gamma$ on $\ell^{2} \Gamma$.

This property originates from Haagerup's seminal paper [9], where it is shown that free groups have what we now call property $\mathrm{RD}$. The explicit definition of property RD is due to Jolissaint [10]; a result from [10] we would like to quote here is the fact that groups of polynomial growth have property RD. As further examples of groups satisfying property RD, we mention: hyperbolic groups ([5]) and, more generally, groups that are relatively hyperbolic to subgroups having property RD ([7); groups acting freely on finite dimensional CAT(0) cube complexes ([2]); cocompact lattices in $\mathrm{SL}_{3}(\mathbb{F})$ for $\mathbb{F}$ a local field $([15])$ and for $\mathbb{F}=\mathbb{R}, \mathbb{C}([1])$; mapping class groups ([1]).

Property RD for a group $\Gamma$ is relevant to the study of its reduced $\mathrm{C}^{*}$-algebra $\mathrm{C}_{\mathrm{r}}^{*} \Gamma$. The first significant use of property RD is the proof by Connes and Moscovici [4] that hyperbolic groups satisfy the Novikov conjecture. A related $K$-theoretic application features in the remarkable work of Lafforgue [12, leading eventually to the proof that hyperbolic groups satisfy the Baum-Connes conjecture ([13, [12]). In another direction, Property RD is used in 8 to show that $\mathrm{C}_{\mathrm{r}}^{*} \Gamma$ has stable rank 1 whenever $\Gamma$ is a torsion-free, non-elementary hyperbolic group.

In this paper, we are interested in quantifying property RD. For $s \geq 0$ consider the following property:

$\left(\mathrm{RD}_{\bullet}^{s}\right)$ There is $C \geq 0$ so that, for all integers $n \geq 0$, we have $\|a\| \leq C(1+n)^{s}\|a\|_{2}$ whenever $a \in \mathbb{C} \Gamma$ is supported on elements of length at most $n$.

Received by the editors August 22, 2009, and, in revised form, November 12, 2009.

2010 Mathematics Subject Classification. Primary 20F99, 22D15, 46E39.

(C)2010 American Mathematical Society

Reverts to public domain 28 years from publication 
Note that the satisfaction of $\left(\mathrm{RD}_{\bullet}^{s}\right)$ does not depend on the choice of word-length for $\Gamma$.

Finite groups satisfy $\left(\mathrm{RD}_{\bullet}^{0}\right)$. Conversely, infinite groups cannot satisfy $\left(\mathrm{RD}_{\bullet}^{0}\right)$; this was first proved by Rajagopalan in 14. Rajagopalan's result was a partial positive answer towards the " $L^{p}$-conjecture", which he formulated around 1960: if $G$ is a locally compact group, and if $L^{p}(G)$ is closed under convolution for some $p \in(1, \infty)$, then $G$ is a compact. The complete resolution of the $L^{p}$-conjecture is due to Saeki [16, almost 30 years after its formulation.

The following question then arises: if an infinite group satisfies $\left(\mathrm{RD}_{\bullet}^{s}\right)$, how small can $s$ be? The main result of this paper answers this question.

Theorem. Let $\Gamma$ be an infinite, finitely generated group. If $\Gamma$ satisfies $\left(\mathrm{RD}_{\bullet}^{s}\right)$, then $s \geq \frac{1}{2}$.

This result is sharp, since $\left(\mathrm{RD}_{\bullet}^{s}\right)$ for $s=\frac{1}{2}$ is satisfied by all virtually- $\mathbb{Z}$ groups.

We do not know how to dismiss the main theorem as being trivial, so we settle for the next best thing: an elementary proof (given in Section 31), inspired by Saeki's solution to the $L^{p}$-conjecture. Rajagopalan's proof from [14, using structural results on so-called $H^{*}$-algebras, does not seem flexible enough to be adaptable here.

It should be pointed out that the main theorem is interesting for infinite torsion groups only, much like the $L^{p}$-conjecture. Indeed, if $\Gamma$ contains an infinite cyclic subgroup, then the main result is a mere observation (cf. Prop. 2.2 and Prop. 2.3). We are therefore accounting here for the possibility that infinite torsion groups with property RD might exist, a possibility which seems completely open at this time.

\section{Preliminaries}

Throughout this paper, groups are assumed to be finitely generated and equipped with a word-length. The choice of word-length is irrelevant for the discussion herein.

2.1. Notation. For two numerical functions $f, g: \mathbb{N} \rightarrow \mathbb{N}$, we write $f(n) \preccurlyeq g(n)$ to mean that there is $C \geq 0$ such that $f(n) \leq C g(n)$ for all $n \in \mathbb{N}$. We write $f(n) \asymp g(n)$ whenever we have both $f(n) \preccurlyeq g(n)$ and $g(n) \preccurlyeq f(n)$; i.e., there are $C \geq c \geq 0$ such that $c g(n) \leq f(n) \leq C g(n)$ for all $n \in \mathbb{N}$.

2.2. A reformulation of property $\mathbf{R D}$. An equivalent definition of property $\mathrm{RD}$ is the following: a group $\Gamma$ is said to satisfy property $\mathrm{RD}$ if there are $C, s \geq 0$ such that $\|a\| \leq C\|a\|_{2, s}$ for all $a \in \mathbb{C} \Gamma$, where

$$
\left\|\sum a_{g} g\right\|_{2, s}:=\left\|\sum a_{g}(1+|g|)^{s} g\right\|_{2}=\sqrt{\sum\left|a_{g}\right|^{2}(1+|g|)^{2 s}} .
$$

Correspondingly, we quantify by considering, for $s \geq 0$, the following property:

$\left(\mathrm{RD}^{s}\right)$ There is $C \geq 0$ such that $\|a\| \leq C\|a\|_{2, s}$ for all $a \in \mathbb{C} \Gamma$.

Informally, $\left(\mathrm{RD}^{s}\right)$ is a "de-localized" version of $\left(\mathrm{RD}_{\bullet}^{s}\right)$ (recall, the latter is a property defined with reference to balls, hence the label $\bullet$ ). The equivalence between the two formulations of property $\mathrm{RD}$ is well-known. The next lemma records this equivalence in a precise fashion.

Lemma 2.1. We have that $\left(\mathrm{RD}^{s}\right)$ implies $\left(\mathrm{RD}_{\bullet}^{s}\right)$, and that $\left(\mathrm{RD}_{\bullet}^{s}\right)$ implies $\left(\mathrm{RD}^{s+\epsilon}\right)$ for all $\epsilon>0$. 
Proof. The first implication is obvious. The second implication is essentially contained in the proof of Proposition 1.2a) in [11] and works as follows. Assume that $\Gamma$ satisfies $\left(\mathrm{RD}_{\bullet}^{s}\right)$. For $n \geq 0$ we let $A_{n}$ be the annulus $\left\{g \in \Gamma: 2^{n}-1 \leq|g|<2^{n+1}-1\right\}$. Then for $a=\sum a_{g} g \in \mathbb{C} \Gamma$ we have:

$$
\begin{aligned}
\left\|\sum a_{g} g\right\| & \leq \sum_{n \geq 0}\left\|\sum_{g \in A_{n}} a_{g} g\right\|^{\left(\mathrm{RD}_{0}^{s}\right)} \leq C \sum_{n \geq 0} 2^{s(n+1)}\left\|\sum_{g \in A_{n}} a_{g} g\right\|_{2} \\
& \leq C \sum_{n \geq 0} 2^{s(n+1)-(s+\epsilon) n}\left\|\sum_{g \in A_{n}} a_{g} g\right\|_{2, s+\epsilon}=2^{s} C \sum_{n \geq 0} 2^{-\epsilon n}\left\|\sum_{g \in A_{n}} a_{g} g\right\|_{2, s+\epsilon} \\
& \leq 2^{s} C\left(\sum_{n \geq 0} 2^{-2 \epsilon n}\right)^{\frac{1}{2}}\left(\sum_{n \geq 0}\left\|\sum_{g \in A_{n}} a_{g} g\right\|_{2, s+\epsilon}^{2}\right)^{\frac{1}{2}}=C^{\prime}(s, \epsilon)\left\|\sum a_{g} g\right\|_{2, s+\epsilon}
\end{aligned}
$$

We conclude that $\Gamma$ satisfies $\left(\mathrm{RD}^{s+\epsilon}\right)$ for each $\epsilon>0$.

In general, $\left(\mathrm{RD}_{\bullet}^{s}\right)$ does not imply $\left(\mathrm{RD}^{s}\right)$; see the following example.

2.3. Groups of polynomial growth. Let $\Gamma$ be a group of polynomial growth. Then its growth function $\gamma$ satisfies $\gamma(n) \asymp n^{d(\Gamma)}$ for some integer $d(\Gamma)$ called the degree of growth of $\Gamma$ (see [6, VII.26 and VII.29]). With this notion at hand, we can state the following explicit description:

Proposition 2.2. Let $\Gamma$ be a group of polynomial growth. Then:

(1) $\Gamma$ satisfies $\left(\mathrm{RD}_{\bullet}^{s}\right)$ if and only if $s \geq \frac{1}{2} d(\Gamma)$;

(2) $\Gamma$ satisfies $\left(\mathrm{RD}^{s}\right)$ if and only if $s>\frac{1}{2} d(\Gamma)$.

Proof. The following fact quantifies a result of Jolissaint [10, Cor.3.1.8] saying that the amenable groups which enjoy property $\mathrm{RD}$ are precisely the polynomial growth groups:

Fact. Let $\Gamma$ be amenable, with growth function $\gamma$. Then $\Gamma$ satisfies $\left(\mathrm{RD}_{\bullet}^{s}\right)$ if and only if $\gamma(n) \preccurlyeq n^{2 s}$.

If $\Gamma$ satisfies $\left(\mathrm{RD}_{\bullet}^{s}\right)$, then $\left\|\chi\left(B_{n}\right)\right\| \leq C(1+n)^{s} \sqrt{\gamma(n)}$, where $\chi\left(B_{n}\right)$ is the characteristic function of the $n$-ball of $\Gamma$. Since $\Gamma$ is amenable, we have that $\|a\|=\|a\|_{1}$ for all $a \in \mathbb{C} \Gamma$ with positive coefficients; for $\chi\left(B_{n}\right)$, we get $\left\|\chi\left(B_{n}\right)\right\|=\gamma(n)$. It follows that $\gamma(n) \leq C^{2}(1+n)^{2 s}$.

Conversely, assume that $\gamma(n) \leq C n^{2 s}$ for some $C>0$. For $a \in \mathbb{C} \Gamma$ supported on elements of length at most $n$, we have $\|a\| \leq$ $\|a\|_{1} \leq \sqrt{\gamma(n)}\|a\|_{2} \leq \sqrt{C}(1+n)^{s}\|a\|_{2}$. Thus $\Gamma$ satisfies $\left(\mathrm{RD}_{\bullet}^{s}\right)$.

The above fact yields Part (1). Most of Part (2) follows by combining Part (1) with Lemma 2.1 the only thing left to check is that $\Gamma$ does not satisfy $\left(\mathrm{RD}^{s}\right)$ at the critical value $s=\frac{1}{2} d(\Gamma)$. Arguing by contradiction, let us assume that $\Gamma$ satisfies $\left(\mathrm{RD}^{s}\right)$ for $s=\frac{1}{2} d(\Gamma)$. Fix an integer $N \geq 0$ and consider

$$
a_{N}=\sum_{1 \leq n \leq N}(1+n)^{-d(\Gamma)} \chi\left(S_{n}\right) \in \mathbb{C} \Gamma,
$$


where $\chi\left(S_{n}\right)$ denotes the characteristic function of the $n$-sphere of $\Gamma$. We have $\left\|a_{N}\right\| \leq C\left\|a_{N}\right\|_{2, s}$, where $C \geq 0$ is independent of $N$. Since

$$
\left\|a_{N}\right\|=\sum_{1 \leq n \leq N}(1+n)^{-d(\Gamma)}\left|S_{n}\right|, \quad\left\|a_{N}\right\|_{2, s}=\sqrt{\sum_{1 \leq n \leq N}(1+n)^{-d(\Gamma)\left|S_{n}\right|}},
$$

we infer that $\sum(1+n)^{-d(\Gamma)}\left|S_{n}\right|$ converges. This is absurd, since $\left|S_{n}\right| \succcurlyeq n^{d(\Gamma)-1}$ (see [6, VII.32]).

2.4. Heredity. We make the following observation, whose easy proof is left to the reader.

Proposition 2.3. Let $\Gamma^{\prime}$ be a subgroup of $\Gamma$.

(1) If $\Gamma$ satisfies $\left(\mathrm{RD}_{\bullet}^{s}\right)$, then $\Gamma^{\prime}$ satisfies $\left(\mathrm{RD}_{\bullet}^{s}\right)$.

(2) Assume that $\Gamma^{\prime}$ has finite index in $\Gamma$. Then $\Gamma$ satisfies $\left(\mathrm{RD}_{\bullet}^{s}\right)$ if and only if $\Gamma^{\prime}$ satisfies $\left(\mathrm{RD}_{\bullet}^{s}\right)$.

This proposition also holds for $\left(\mathrm{RD}^{s}\right)$ instead of $\left(\mathrm{RD}_{\bullet}^{s}\right)$.

\section{Proof of MAIN THEOREM}

This section contains the proof of our main result. We start off with two lemmas which are free of any RD assumption. In what follows, products of functions on $\Gamma$ are convolution products, and inequalities are in the pointwise sense.

Lemma 3.1. For all $n, k \geq 1$ we have $\chi\left(B_{n}\right) \chi\left(B_{n+k}\right) \geq\left|B_{n}\right| \chi\left(B_{k}\right)$.

Proof. The coefficient of each $h \in B_{k}$ is at least $\left|B_{n}\right|$, since $g^{-1} h \in B_{n+k}$ whenever $g \in B_{n}$.

For $r \geq 1$ and $\alpha>0$, consider the following formal sum:

$$
Z_{r}(\alpha)=\sum_{k \geq 1} k^{-\alpha} \frac{\chi\left(B_{r k}\right)}{\left\|\chi\left(B_{r k}\right)\right\|_{2}}
$$

Lemma 3.2. Let $\alpha, \beta>0$ with $\alpha+\beta>1$. Then $Z_{r}(\alpha) Z_{r}(\beta) \geq \frac{1}{\alpha+\beta-1} Z_{r}(\alpha+\beta-1)$.

Proof. Lemma 3.1, together with the obvious inequality

$$
\left\|\chi\left(B_{r(j+k)}\right)\right\|_{2} \leq\left\|\chi\left(B_{r j}\right)\right\|_{2}\left\|\chi\left(B_{r k}\right)\right\|_{2},
$$

gives

$$
\frac{\chi\left(B_{r k}\right)}{\left\|\chi\left(B_{r k}\right)\right\|_{2}} \frac{\chi\left(B_{r(j+k)}\right)}{\left\|\chi\left(B_{r(j+k)}\right)\right\|_{2}} \geq \frac{\left\|\chi\left(B_{r k}\right)\right\|_{2}^{2} \chi\left(B_{r j}\right)}{\left\|\chi\left(B_{r k}\right)\right\|_{2}\left\|\chi\left(B_{r(j+k)}\right)\right\|_{2}} \geq \frac{\chi\left(B_{r j}\right)}{\left\|\chi\left(B_{r j}\right)\right\|_{2}}
$$

for all $j, k \geq 1$. Therefore

$$
\begin{aligned}
Z_{r}(\alpha) Z_{r}(\beta) & \geq \sum_{j \geq 1} \sum_{k \geq 1} k^{-\alpha}(j+k)^{-\beta} \frac{\chi\left(B_{r k}\right)}{\left\|\chi\left(B_{r k}\right)\right\|_{2}} \frac{\chi\left(B_{r(j+k)}\right)}{\left\|\chi\left(B_{r(j+k)}\right)\right\|_{2}} \\
& \geq \sum_{j \geq 1}\left(\sum_{k \geq 1}(j+k)^{-\alpha-\beta}\right) \frac{\chi\left(B_{r j}\right)}{\left\|\chi\left(B_{r j}\right)\right\|_{2}} .
\end{aligned}
$$


Since for each $j \geq 1$ we have

$$
\sum_{k \geq 1}(j+k)^{-(\alpha+\beta)}>\int_{j}^{\infty} x^{-(\alpha+\beta)} d x=\frac{j^{-(\alpha+\beta-1)}}{\alpha+\beta-1},
$$

the desired inequality follows.

We now come to the proof of

Theorem 3.3. If $\Gamma$ is an infinite group satisfying $\left(\mathrm{RD}_{\bullet}^{s}\right)$, then $s \geq \frac{1}{2}$.

Proof. Assume, by way of contradiction, that $\Gamma$ satisfies $\left(\mathrm{RD}_{\bullet}^{s}\right)$ for some $s \in\left[0, \frac{1}{2}\right)$. Using Lemma 3.1 and $\left(\mathrm{RD}_{\bullet}^{s}\right)$, we have

$$
\begin{aligned}
\left\|\chi\left(B_{r}\right)\right\|_{2}^{2}\left\|\chi\left(B_{r k}\right)\right\|_{2} & =\left\|\left|B_{r}\right| \chi\left(B_{r k}\right)\right\|_{2} \leq\left\|\chi\left(B_{r}\right) \chi\left(B_{r(k+1)}\right)\right\|_{2} \\
& \leq\left\|\chi\left(B_{r}\right)\right\|\left\|\chi\left(B_{r(k+1)}\right)\right\|_{2} \leq C(1+r)^{s}\left\|\chi\left(B_{r}\right)\right\|_{2}\left\|\chi\left(B_{r(k+1)}\right)\right\|_{2} ;
\end{aligned}
$$

that is,

$$
\left\|\chi\left(B_{r(k+1)}\right)\right\|_{2} \geq \frac{\left\|\chi\left(B_{r}\right)\right\|_{2}}{C(1+r)^{s}}\left\|\chi\left(B_{r k}\right)\right\|_{2}
$$

for all $r, k \geq 1$. Fix $r \geq 1$ such that $\left\|\chi\left(B_{r}\right)\right\|_{2} \geq 2 C(1+r)^{s}$, a choice made possible by the fact that the volume growth of $\Gamma$ is at least linear. Then $\left\|\chi\left(B_{r(k+1)}\right)\right\|_{2} \geq$ $2\left\|\chi\left(B_{r k}\right)\right\|_{2}$ for all $k \geq 1$.

Next, we claim that $Z_{r}(\alpha) \in \ell^{2} \Gamma$ if and only if $\alpha>\frac{1}{2}$. To show this, we compare $\left\|Z_{r}(\alpha)\right\|_{2}^{2}$ against $\sum k^{-2 \alpha}$. One bound holds in general:

$$
\left\|Z_{r}(\alpha)\right\|_{2}^{2} \geq \sum_{k=l \geq 1} k^{-\alpha} l^{-\alpha}\left\langle\frac{\chi\left(B_{r k}\right)}{\left\|\chi\left(B_{r k}\right)\right\|_{2}}, \frac{\chi\left(B_{r l}\right)}{\left\|\chi\left(B_{r l}\right)\right\|_{2}}\right\rangle=\sum_{k \geq 1} k^{-2 \alpha}
$$

For the other bound, we write

$$
\begin{aligned}
\left\|Z_{r}(\alpha)\right\|_{2}^{2} & \leq 2 \sum_{l \geq k \geq 1} k^{-\alpha} l^{-\alpha}\left\langle\frac{\chi\left(B_{r k}\right)}{\left\|\chi\left(B_{r k}\right)\right\|_{2}}, \frac{\chi\left(B_{r l}\right)}{\left\|\chi\left(B_{r l}\right)\right\|_{2}}\right\rangle \\
& =2 \sum_{k \geq 1}\left(\sum_{l \geq k} \frac{l^{-\alpha}}{\left\|\chi\left(B_{r l}\right)\right\|_{2}}\right) k^{-\alpha}\left\|\chi\left(B_{r k}\right)\right\|_{2} .
\end{aligned}
$$

From $\left\|\chi\left(B_{r(l+1)}\right)\right\|_{2} \geq 2\left\|\chi\left(B_{r l}\right)\right\|_{2}$ we deduce that

$$
\frac{(l+1)^{-\alpha}}{\left\|\chi\left(B_{r(l+1)}\right)\right\|_{2}}<\frac{1}{2} \frac{l^{-\alpha}}{\left\|\chi\left(B_{r l}\right)\right\|_{2}}
$$

hence

$$
\sum_{l \geq k} \frac{l^{-\alpha}}{\left\|\chi\left(B_{r l}\right)\right\|_{2}}<2 \frac{k^{-\alpha}}{\left\|\chi\left(B_{r k}\right)\right\|_{2}}
$$

which, in turn, implies $\left\|Z_{r}(\alpha)\right\|_{2}^{2}<4 \sum_{k \geq 1} k^{-2 \alpha}$. The claim is proved.

Pick $t$ such that $s<t<\frac{1}{2}$. Also, pick $\alpha, \beta$ such that the following are satisfied:

$$
\alpha>\frac{1}{2}+t, \quad \beta>\frac{1}{2}, \quad \alpha+\beta-1 \leq \frac{1}{2}
$$


On the one hand, $Z_{r}(\alpha+\beta-1)$ is not in $\ell^{2} \Gamma$. On the other hand, $Z_{r}(\alpha-t)$ and $Z_{r}(\beta)$ are in $\ell^{2} \Gamma$. From

$$
\begin{aligned}
\left\|Z_{r}(\alpha)\right\|_{2, t} & \leq\left\|\sum_{k \geq 1} k^{-\alpha}(1+r k)^{t} \frac{\chi\left(B_{r k}\right)}{\left\|\chi\left(B_{r k}\right)\right\|_{2}}\right\|_{2} \\
& \leq\left\|\sum_{k \geq 1} k^{-\alpha}(2 r k)^{t} \frac{\chi\left(B_{r k}\right)}{\left\|\chi\left(B_{r k}\right)\right\|_{2}}\right\|_{2}=(2 r)^{t}\left\|Z_{r}(\alpha-t)\right\|_{2}
\end{aligned}
$$

and the fact that $\left(\mathrm{RD}^{t}\right)$ holds (Lemma 2.1), we deduce that $Z_{r}(\alpha)$ is a bounded operator on $\ell^{2} \Gamma$. Then $Z_{r}(\alpha) Z_{r}(\beta)$ is in $\ell^{2} \Gamma$, so $Z_{r}(\alpha+\beta-1)$ is in $\ell^{2} \Gamma$ by Lemma3.2. This contradiction ends the proof.

\section{FinAl REMARKS}

According to Lemma 2.1. we have

$$
\inf \left\{s: \Gamma \text { satisfies }\left(\operatorname{RD}^{s}\right)\right\}=\inf \left\{s: \Gamma \text { satisfies }\left(\operatorname{RD}_{\bullet}^{s}\right)\right\} \text {; }
$$

we denote this quantity by $\operatorname{rd}(\Gamma)$ and we call it the $R D$-degree of $\Gamma$. By definition, $\operatorname{rd}(\Gamma)$ is finite precisely when $\Gamma$ has property $\mathrm{RD}$. Observe that $\operatorname{rd}(\Gamma)$ is independent of the choice of word-length for $\Gamma$.

In terms of the RD-degree, our discussion can be summarized as follows:

- if $\Gamma$ is infinite, then $\operatorname{rd}(\Gamma) \geq \frac{1}{2}$; if $\Gamma$ is finite, then $\operatorname{rd}(\Gamma)=0$;

- if $\Gamma$ has polynomial growth, then $\operatorname{rd}(\Gamma)=\frac{1}{2} d(\Gamma)$, where $d(\Gamma)$ denotes the growth degree of $\Gamma$;

- if $\Gamma^{\prime}$ is a subgroup of $\Gamma$, then $\operatorname{rd}\left(\Gamma^{\prime}\right) \leq \operatorname{rd}(\Gamma)$;

- if $\Gamma^{\prime}$ and $\Gamma$ are commensurable, then $\operatorname{rd}\left(\Gamma^{\prime}\right)=\operatorname{rd}(\Gamma)$.

These properties suggest that $2 \operatorname{rd}(\cdot)$ can be thought of as a dimension function on finitely generated groups. Computing the RD-degree of other groups would be, of course, interesting in this regard. We single out the following.

Problem. Compute $\mathrm{rd}\left(\mathrm{F}_{r}\right)$, where $\mathrm{F}_{r}$ denotes the free group of rank $r \geq 2$.

Note that the answer is independent of $r$. It is known that $1 \leq \operatorname{rd}\left(\mathrm{F}_{r}\right) \leq \frac{3}{2}$ (Haagerup's estimates from [9] yield the upper bound, whereas the lower bound is a consequence of Cohen's computations from [3]).

We close by reminding the reader that the following question is open: Are there infinite, finitely generated torsion groups which enjoy property RD?

\section{ACKNOWLEDGMENTS}

The author thanks Gennadi Kasparov and Guoliang Yu for financial support during the summer of 2009. He also thanks the referee for some useful comments.

\section{REFERENCES}

[1] J. A. Behrstock, Y. N. Minsky: Centroids and the rapid decay property in mapping class groups, arXiv:0810.1969

[2] I. Chatterji, K. Ruane: Some geometric groups with rapid decay, Geom. Funct. Anal. 15 (2005), no. 2, 311-339. MR2153902 (2006d:20070)

[3] J. M. Cohen: Operator norms on free groups, Boll. Un. Mat. Ital. B (6) 1 (1982), no. 3, 1055-1065. MR683492 (85d:22011)

[4] A. Connes, H. Moscovici: Cyclic cohomology, the Novikov conjecture and hyperbolic groups, Topology 29 (1990), no. 3, 345-388. MR1066176 (92a:58137) 
[5] P. de la Harpe: Groupes hyperboliques, algèbres d'opérateurs et un théorème de Jolissaint, C. R. Acad. Sci. Paris Sér. I Math. 307 (1988), no. 14, 771-774. MR972078 (90d:22005)

[6] P. de la Harpe: Topics in geometric group theory, Chicago Lectures in Mathematics, University of Chicago Press, 2000. MR.1786869 (2001i:20081)

[7] C. Druţu, M. Sapir: Relatively hyperbolic groups with rapid decay property, Int. Math. Res. Not. (2005), no. 19, 1181-1194. MR2147058 (2006d:20077)

[8] K. J. Dykema, P. de la Harpe: Some groups whose reduced $C^{*}$-algebras have stable rank one, J. Math. Pures Appl. (9) 78 (1999), no. 6, 591-608. MR1708667 (2000f:46070)

[9] U. Haagerup: An example of a nonnuclear $C^{*}$-algebra, which has the metric approximation property, Invent. Math. 50 (1978/79), no. 3, 279-293. MR520930 (80j:46094)

[10] P. Jolissaint: Rapidly decreasing functions in reduced $C^{*}$-algebras of groups, Trans. Amer. Math. Soc. 317 (1990), no. 1, 167-196. MR.943303 (90d:22006)

[11] V. Lafforgue: A proof of property $(R D)$ for cocompact lattices of $\mathrm{SL}(3, \mathbb{R})$ and $\mathrm{SL}(3, \mathbb{C})$, J. Lie Theory 10 (2000), no. 2, 255-267. MR.1774859 (2001g:22020)

[12] V. Lafforgue: K-théorie bivariante pour les algèbres de Banach et conjecture de BaumConnes, Invent. Math. 149 (2002), no. 1, 1-95. MR.1914617(2003d:19008)

[13] I. Mineyev, G. Yu: The Baum-Connes conjecture for hyperbolic groups, Invent. Math. 149 (2002), no. 1, 97-122. MR.1914618 (2003f:20072)

[14] M. Rajagopalan: On the $L^{p}$-space of a locally compact group, Colloq. Math. 10 (1963), 49-52. MR0148792 (26:6297)

[15] J. Ramagge, G. Robertson, T. Steger: A Haagerup inequality for $\widetilde{A}_{1} \times \widetilde{A}_{1}$ and $\widetilde{A}_{2}$ buildings, Geom. Funct. Anal. 8 (1998), no. 4, 702-731. MR1633983 (99j:20046)

[16] S. Saeki: The $L^{p}$-conjecture and Young's inequality, Illinois J. Math. 34 (1990), no. 3, 614627. MR.1053566 (91j:43005)

Department of Mathematics, Vanderbilt University, Nashville, Tennessee 37240

Current address: Department of Mathematics and Statistics, University of Victoria, Victoria, BC, Canada V8W 3R4 